\title{
Indian hedgehog stimulates periarticular chondrocyte differentiation to regulate growth plate length independently of PTHrP
}

\author{
Tatsuya Kobayashi, ${ }^{1}$ Desi W. Soegiarto, ${ }^{2}$ Yingzi Yang, ${ }^{3}$ Beate Lanske,,2,4 \\ Ernestina Schipani, ${ }^{1}$ Andrew P. McMahon, ${ }^{5}$ and Henry M. Kronenberg ${ }^{1}$
}

\begin{abstract}
${ }^{1}$ Endocrine Unit, Massachusetts General Hospital, Boston, Massachusetts, USA. ${ }^{2}$ Molecular Endocrinology, Max-Planck-Institute for Biochemistry, Martinsried, Germany. ${ }^{3}$ Developmental Genetics Section, Genetic Disease Research Branch, National Human Genome Research Institute, Bethesda, Maryland, USA. ${ }^{4}$ Department of Oral and Developmental Biology, The Forsyth Institute, Boston, Massachusetts, USA. ${ }^{5}$ Department of Molecular and Cellular Biology, Harvard University, Cambridge, Massachusetts, USA.
\end{abstract}

\begin{abstract}
In the developing growth plate, periarticular chondrocytes proliferate, differentiate into columnar chondrocytes, and then further differentiate into postmitotic hypertrophic chondrocytes. Parathyroid hormone-related (PTH-related) protein (PTHrP), regulated by Indian hedgehog (Ihh), prevents premature hypertrophic differentiation, thereby maintaining the length of columns. Ihh regulates cartilage development through PTHrP-independent pathways as well. Here we show that Ihh stimulates differentiation of periarticular to columnar chondrocytes (periarticular chondrocyte differentiation) and thereby regulates the length of columns independently of PTHrP. Mosaic ablation of the PTH/PTHrP receptor in the growth plate caused upregulation of Ihh action, PTHrP upregulation, acceleration of periarticular chondrocyte differentiation, and elongation of the columnar region. Decreasing Ihh action in these mice reduced elongation of columns, whereas decreasing PTHrP showed only a modest effect on column length. Overexpression of Ihh caused PTHrP upregulation, elongation of columns, and acceleration of periarticular chondrocyte differentiation. PTHrP heterozygosity in this model had a minimal effect on the elongation of columns. Moreover, the elongation of columns and stimulation of periarticular chondrocyte differentiation in these models were still observed when PTHrP signaling was maintained so that it remained constant. These results demonstrate that Ihh acts on periarticular chondrocytes to stimulate their differentiation, thereby regulating the columnar cell mass independently of PTHrP.
\end{abstract}

\section{Introduction}

In the late embryonic mouse growth plate, 3 morphologically distinct groups of chondrocytes form an orderly structure: round periarticular chondrocytes, flat column-forming chondrocytes, and hypertrophic chondrocytes. Periarticular chondrocytes proliferate and differentiate into flat, columnar chondrocytes that vigorously proliferate while forming columns. Eventually columnar chondrocytes stop proliferating and differentiate into hypertrophic chondrocytes. Coordination of these differentiation processes to regulate chondrocyte mass and cartilage structure is critical for normal development and growth of bone. Parathyroid hormonerelated (PTH-related) protein (PTHrP) and Indian hedgehog (Ihh) are important regulators of cartilage development. PTHrP is produced by periarticular chondrocytes and perichondrial cells on the articular surface. Both PTHrP-null ( $P$ THrP $P^{-/} /$Pthlh, Mouse Genome Informatics [MGI]; http://www.informatics.jax.org/) and PTH/ PTHrP receptor-null (PPR-null) $\left(P P R^{-/-} / P t h r, M G I\right)$ mice exhibit

\footnotetext{
Nonstandard abbreviations used: Bapx1, bagpipe homeobox gene 1 homolog; $c a P P R$, constitutively active mutant of PPR; Col2, type II collagen; ColX, type X collagen; $c-P P R$, constant growth plate PPR signaling; exo-PTHrP, exogenous PTHrP; flpe, enhanced FLP recombinase; frt, FLP recombination target; Ihh, Indian hedgehog; I hh-Bg, Ihb bigenic; MGI, Mouse Genome Informatics; neo-tk, neomycin resistant gene-thymidine kinase gene; Ost-Cre, Cre driven by an osteocalcin promoter; PPR, PTH/PTHrP receptor; Ptc, patched 1; PTH, parathyroid hormone; PTHrP, PTH-related protein; Shh, sonic hedgehog; Smo, smoothened.

Conflict of interest: The authors have declared that no conflict of interest exists.

Citation for this article: J. Clin. Invest. 115:1734-1742 (2005).

doi:10.1172/JCI24397.
}

premature hypertrophic differentiation of chondrocytes, causing a variable reduction in the number of periarticular chondrocytes and a severe reduction in the number of columnar chondrocytes $(1,2)$. PTHrP overexpression or expression of a constitutively active PPR in the growth plate suppresses the initial hypertrophic differentiation $(3,4)$. Based on these findings, PTHrP has been thought to regulate the length of the columnar region by suppressing terminal differentiation into postmitotic hypertrophic chondrocytes.

Ihh, predominantly produced by prehypertrophic and hypertrophic chondrocytes, binds to membrane protein patched 1 (Ptc), which suppresses the activity of smoothened (Smo) in the absence of hedgehog ligands. Upon hedgehog binding to Ptc, Smo is activated. Smo activation leads to transcriptional regulation of hedgehog target genes, including Ptc $(5,6)$. Therefore, Ptc expression principally correlates with hedgehog action in vivo. The upregulation of Ptc as well as hedgehog-interacting protein-1 (Hip-1/ Hhip, MGI), after activation of hedgehog signaling, limits hedgehog ligand diffusion. In cartilage, PTHrP expression is dependent upon and stimulated by Ihh $(7,8)$. I $h b$-null mice and mice with conditional ablation of Smo show reduced chondrocyte proliferation and loss of PTHrP expression in cartilage $(8,9)$. Moreover, analysis of $I h h$-null mice expressing a constitutively active mutant of PPR (caPPR) showed that the proliferation defect in $I h b$-null mice was independent of PTHrP signaling (8-10).

PPR signaling and Ihh signaling form a negative feedback loop that controls fetal growth plate development (11); PPR signaling is required to maintain the columnar region that separates the 

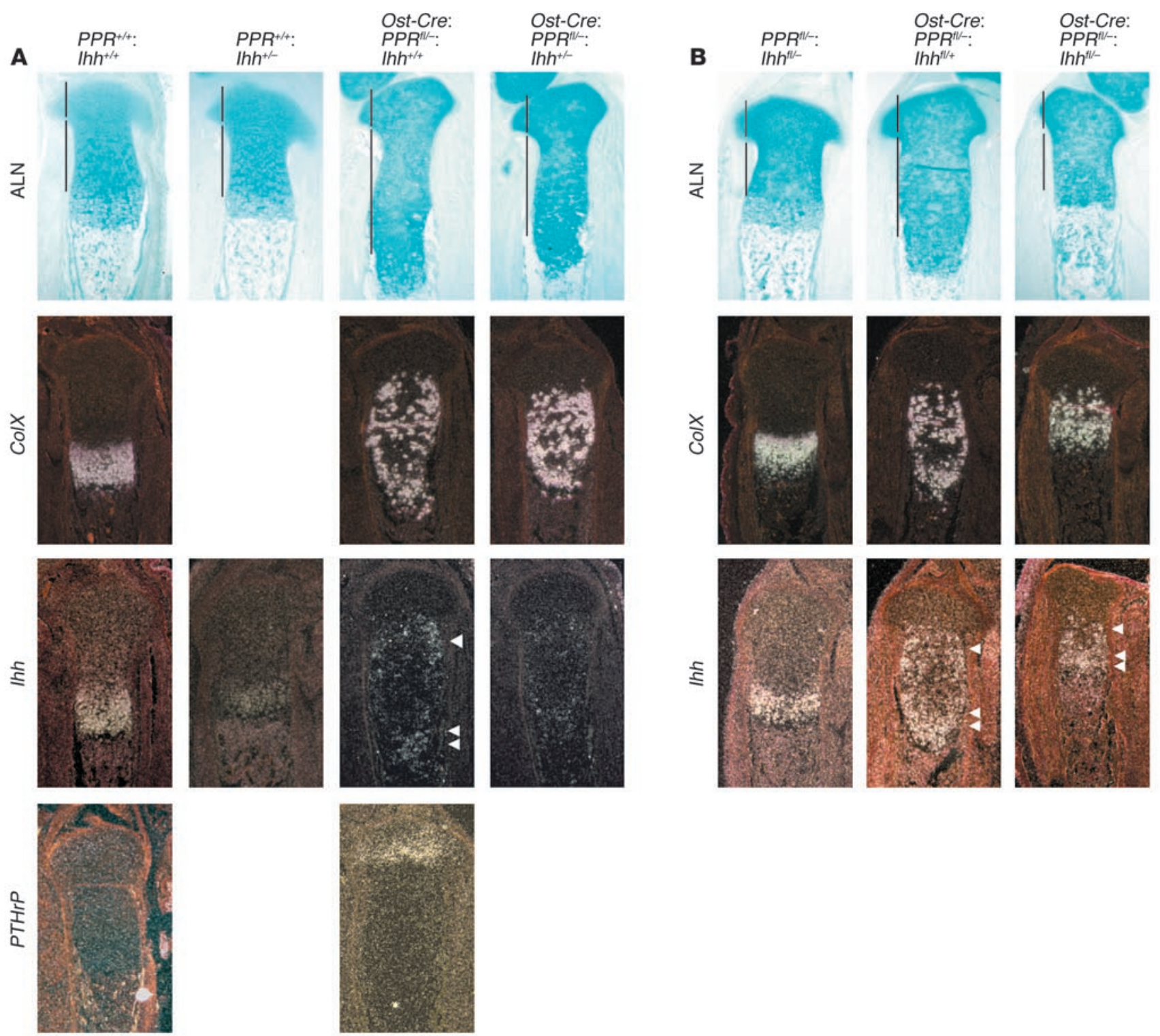

Figure 1

Effect of Ihh reduction in mice with conditional mosaic ablation of the PPR. (A) The growth plate expansion in conditional PPR-mosaic mice (OstCre:PPR ${ }^{f l-}$ ) was partially reduced by Ihh heterozygosity. Ectopic hypertrophic differentiation due to loss of the functional PPR is shown by ectopic expression of the ColX mRNA (ColX; Col10a; MGI) and Ihh. Ihh heterozygosity reduced the Ihh expression level in both ectopic (arrowhead) and eutopic (double arrowhead) hypertrophic chondrocytes. The length of the growth plate (distance between the articular end and the interface between cartilage and bone marrow) was significantly different between Ost-Cre:PPR $R^{f l-}: I h h^{+/+}$and Ost-Cre:PPR ${ }^{f l /}: I h h^{+/-}$mice $\left(100 \% \pm 0.78 \% \mathrm{Ihh} h^{+/+}\right.$versus $93 \% \pm 1.38 \% \mathrm{Ihh^{+/- }}$; littermates were compared, $n=3$ each group; $\left.P<0.05\right)$. There was also modest shortening of the growth plate in $P P R^{+/+}$: $I h h^{+/-}$compared with $P P R^{+/+}: I h h^{+/+}$mice. Vertical lines represent the periarticular and columnar regions. ALN, Alcian blue staining. (B) Conditional double mosaic ablation of the PPR and Ihh genes. The PPR floxed gene and Ihh-floxed gene were combined with the Ost-Cre transgene. In both PPR single mosaic (Ost-Cre:PPR $f / /-: I h h^{f /+}$ ) and PPR/lhh-double-mosaic (Ost-Cre:PPR $R^{f / /}: I h h^{f / /}$ ) growth plates, ectopic hypertrophic differentiation indicated by ColX mRNA (CoIX) expression occurred whereas the level of ectopic lhh mRNA (arrowheads) was reduced compared with that of the eutopic Ihh mRNA expression (double arrowheads). This reduction in Ihh mRNA expression accompanied reduction of growth plate expansion.

domain predominantly expressing Ihh (the prehypertrophic region) from that expressing PTHrP (the periarticular region), thereby delaying the differentiation of cells that synthesize Ihh. Ihh then stimulates production of PTHrP in the periarticular domain and also stimulates chondrocyte proliferation $(8,9)$. Analysis of chimeric mice comprising wild-type cells and $P P R$-null cells demonstrated that the production of Ihh close to the periarticular region by cells missing the PPR leads to an increase in PTHrP production and to an elongation of columns of wild-type chondrocytes $(12,13)$.

Correlation between PTHrP upregulation and elongation of the growth plate was also seen in multiple mouse models with abnormalities in PPR signaling in which Ihh action in the periarticular region was upregulated (14). In these mice, we also found that periarticular chondrocyte differentiation was accelerated. 


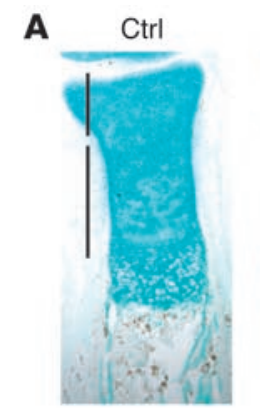

B

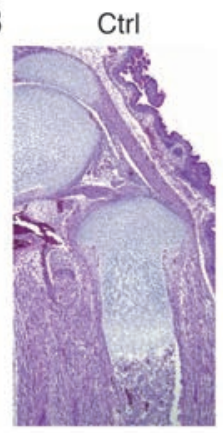

Ihh-Bg
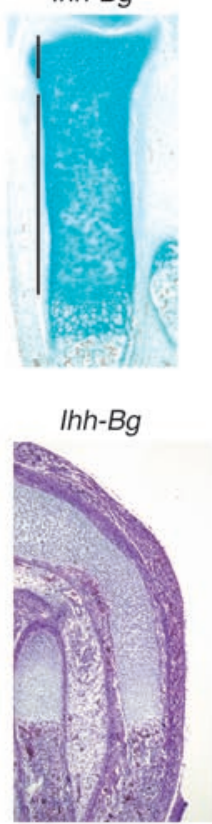
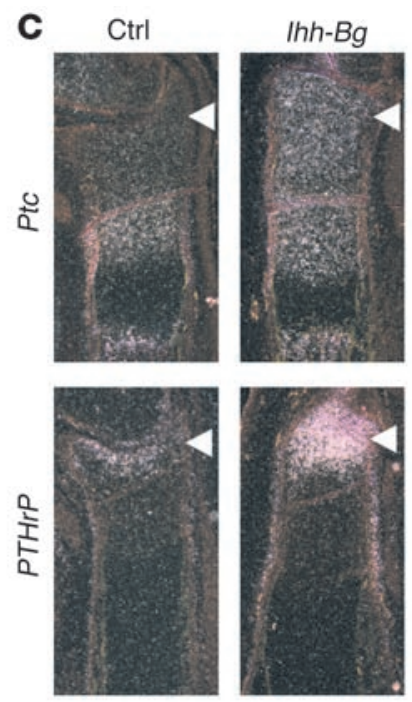

E
D

Ctrl
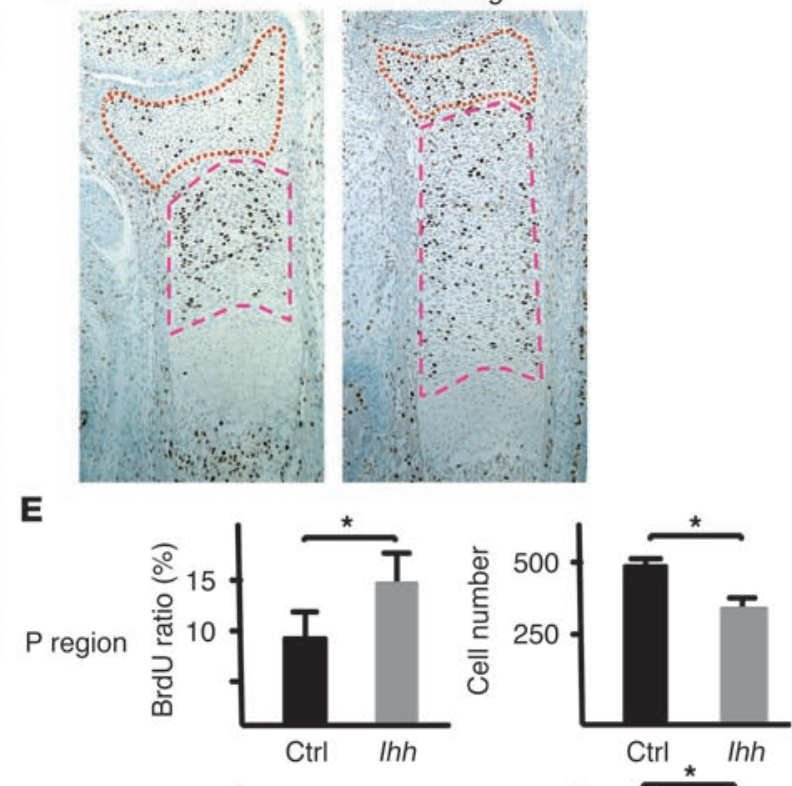

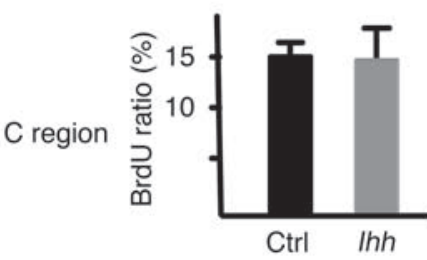

$\mathrm{Ihh}-\mathrm{Bg}$

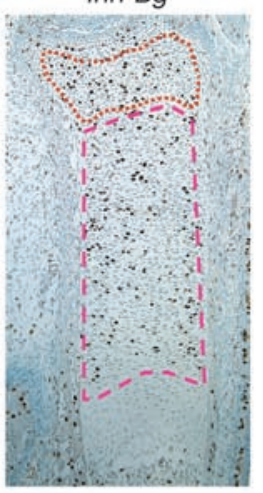

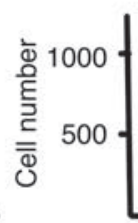

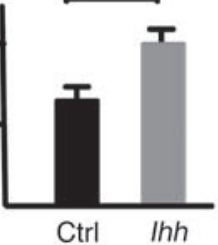

Figure 2

Ih $\mathrm{h}$ overexpression in the growth plate causes growth plate expansion and acceleration of periarticular chondrocyte differentiation. (A) Alcian blue staining showed that $/ \mathrm{h} h$ overexpression in the growth plate by the Col2-GAL4:UAS-clhh bigenic system (Ihh-Bg) caused longitudinal elongation of the growth plate of the E17.5 distal tibia. Col2-GAL4 single-transgenic mice were used as the control (Ctrl). (B) The distal growth plate of the tibia was analyzed due to the knee joint fusion of $\mathrm{Ihh}-\mathrm{Bg}$. (C) Ptc and PTHrP upregulation in the periarticular region of the Ihh-Bg growth plate (arrowheads). ( $\mathbf{D}$ and $\mathbf{E})$ Cell number was reduced despite increased proliferation in the periarticular region of $I h h$ - $B g$ mice. Three mice in each group were analyzed. Sections representing the mid-cross-plane of the tibia were selected for BrdU staining (D) and cell number counting (D and E). Cell number in each region was counted on BrdU/H\&E-stained sections and expressed as BrdU-positive cells/total cells in the periarticular (P) or columnar $(C)$ regions of each section. Red and magenta dotted lines indicate the periarticular and columnar regions, respectively. (E) Despite an approximately $50 \%$ increase in BrdU-labeling ratio in the periarticular region of $/ \mathrm{h} h$ - $\mathrm{Bg}$ mice, the cell number was smaller than that in control mice by approximately $30 \%$. In contrast, the cell number in the columnar (D) region of $/ \mathrm{hh}$ - $\mathrm{Bg}$ mice was approximately $30 \%$ greater, whereas the BrdU labeling ratio was similar to that of control mice. $n=3$ in each group; ${ }^{*} P<0.05$.

Based on the association between Ihh action in periarticular chondrocytes and acceleration in differentiation of periarticular into columnar chondrocytes, we hypothesized that Ihh stimulates periarticular chondrocyte differentiation (periarticular to columnar) to increase the number of columnar chondrocytes and the length of the columnar region, while PTHrP is required for prevention of premature hypertrophic differentiation. In this report, we provide evidence that Ihh stimulates periarticular chondrocyte differentiation to regulate the length of the columnar region and that lengthening of the columnar region occurs even in the absence of PTHrP upregulation.

\section{Results}

Upregulation of Ihb action contributes to growth-plate phenotypes of mice with mosaic ablation of $P P R$ signaling. We examined the effect of upregulation of Ihh action on growth-plate length in mice with PPR-mosaic ablation. In PPR-mosaic mice in which the floxed PPR genes are disrupted in some proliferating chondrocytes (Cre driven by an osteocalcin promoter:PPR $f l /-$ mice [Ost-Cre:PPR $f l-$ mice]) (14), chondrocytes missing PPR signaling prematurely differentiate into hypertrophic chondrocytes in the columnar region, as shown by type X collagen (ColX) and Ibh expression (Figure 1A). This ectopic expression of $I h b$ causes upregulation of Ihh action in the periarticular region, as shown by the increase in Ptc and PTHrP expression (Figure 1A and data not shown). The extent of growth plate elongation of these PPR-mosaic mice (shown by Alcian blue staining) was reduced in an $\mathrm{Ihh}^{+/-}$background $\left(100 \% \pm 0.78 \% \mathrm{Ihh}^{+/+}\right.$vs. $93 \% \pm 1.38 \%$ $\mathrm{Ihh}^{+/-} ; n=3$ in each group; $P<0.05$ ) (Figure 1A). We previously found that upregulation of Ihh action in the periarticular region was associated with accelerated differentiation of periarticular chondrocytes into columnar chondrocytes. This finding was based on an increase in the BrdU-labeling ratio despite smaller than normal sizes of periarticular regions in mice with PPR-mosaic ablation and in PPR-hypomorphic mice (14). We hypothesized, therefore, that BrdU-labeling ratios in periarticular chondrocytes might be lower in $\mathrm{Ihb}^{+/-}$than in $\mathrm{I} \mathrm{h}^{+/+}$backgrounds. We were, however, unable to show statistical differences either in BrdU-labeling ratio or in the

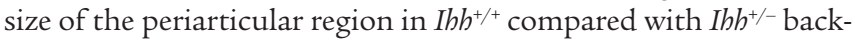
grounds, even when littermates were compared (data not shown). 

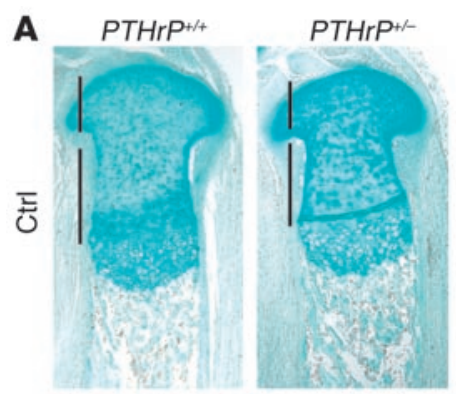

B
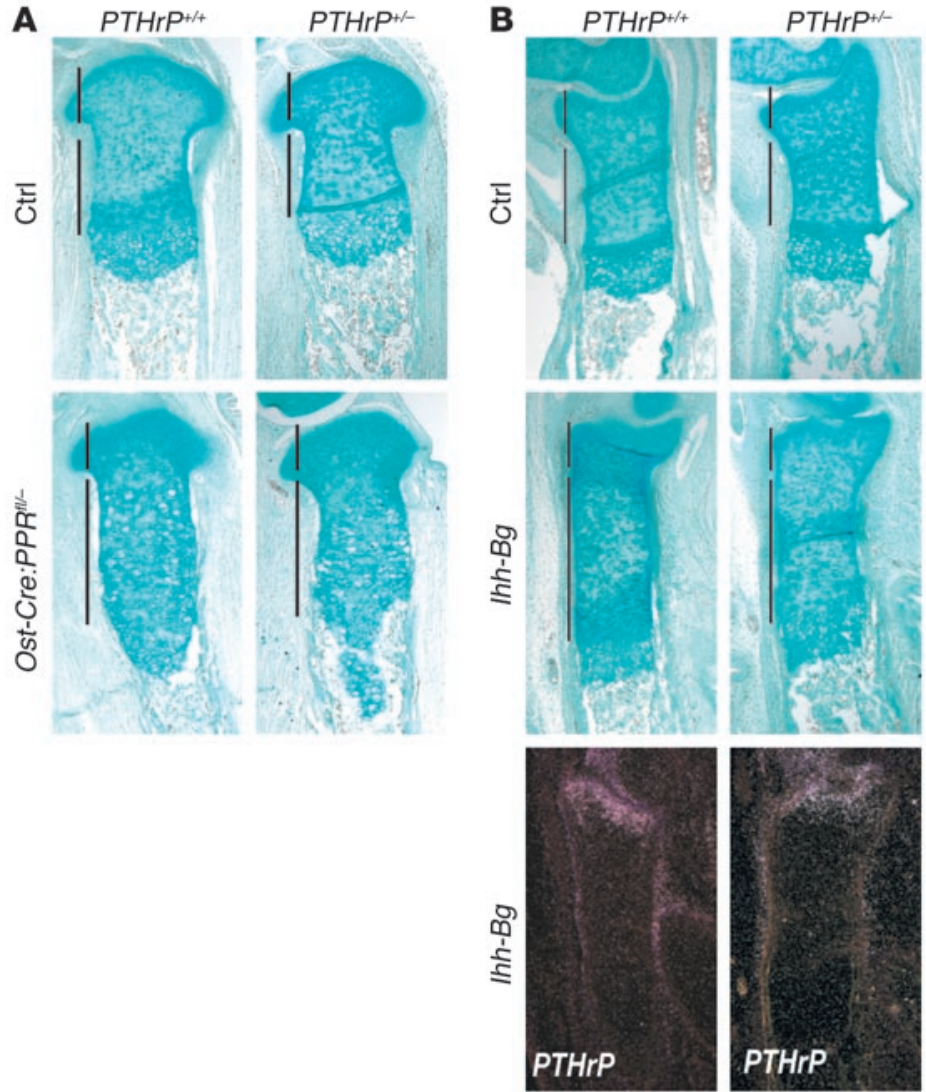

Figure 3

Effect of PTHrP heterozygosity on the length of the growth plate. Alcian blue-stained growth plates of E17.5 proximal tibiae from wild-type and E16.5 proximal tibiae from Ost-Cre:PPR $R^{f l-}$ mice $(\mathbf{A})$ and $\mathrm{E} 16.5$ distal tibiae from control (Col2-GAL4) and Col2-GAL4:UAS-clhh (Ihh-Bg) mice (B) in $\mathrm{PPR}^{+/+}$and $P P R^{+/-}$backgrounds are shown. In situ hybridization confirmed a reduction in PTHrP expression in Ihh-Bg:PTHrP+/- mice. Littermates were compared. Growth plates of $P P R^{+/-}$mice were shorter than those of controls by approximately $8 \%$ regardless of other genetic modifications.

In the columnar region as well, we did not find differences in BrdUlabeling ratio between ${\mathrm{I} h b^{+/+}}$and $\mathrm{I} b b^{+/-}$mice (data not shown). It appears that the effects of Ihh heterozygosity on chondrocyte proliferation are too small to measure accurately at any one moment in time, given current methods, but that these small changes might, over time, lead to substantial morphologic changes.

We further ablated $I h b$ simultaneously with $P P R$ in a mosaic fashion using a floxed $I h b$ conditional allele (Figure 1B). The elongation of the columnar region in $P P R$-single-mosaic mice (Ost-Cre:PPR ${ }^{f l-}$ : $I h h^{f l+}$ ) was no longer observed in the double mosaic mice (Ost-Cre: $\left.P P R^{f l-:}: I h h^{f /-}\right)$, demonstrating that Ihh was responsible for the elongation of the columnar region. Because we were unable to obtain multiple double mosaic mice in single litters, statistical analysis of the BrdU-labeling index in the periarticular region was not possible. Although the identity of the mechanism whereby upregulated Ihh action in PPR-mosaic mice causes growth plate elongation is not clear from this experiment, these results demonstrate the causative role of ectopic Ihh in growth plate elongation in the mosaic mice.

Ibh overexpression causes acceleration of periarticular chondrocyte differentiation. In order to investigate further the effect of Ihh overexpression in chondrocytes, chick-Ihb $(c I h b)$ was overexpressed in the growth plate using the GAL4/UAS binary system. In this system, a type II collagen (Col2) promoter indirectly drives expression of cIhb $(9,15)$. These mice exhibit diverse developmental defects, including acranium, facial malformations, and fusion of knee joints (Figure 2B). Because of the distortion and fusion of growth plates of the proximal tibia and distal femur, we examined instead the distal tibia, in which normal morphology was relatively well preserved. At E17.5, $I h b$ bigenic $(I h b-B g)$ mice showed longitudinal expansion of the growth plate while the width of the bone was diminished (Figure 2A). Upregulation of Ptc and PTHrP expression in the periarticular region indicated an increase in hedgehog signaling (Figure 2C). The BrdU-labeling assay revealed that the proliferation of periarticular chondrocytes (Figure 2E) was significantly increased in $I b h-B g$ mice despite a decrease in the number of periarticular chondrocytes (Figure 2, D and E). The increase in BrdUlabeling ratio and decrease in cell number in the periarticular regions were observed in multiple stages of late fetal development (E16.5, E17.5, and E18.5; data not shown). In contrast, the proliferation rate in the columnar region (Figure $2 \mathrm{E}$ ) did not differ statistically between the genotypes, whereas the number of columnar chondrocytes was significantly greater in $\mathrm{Ibh}-\mathrm{Bg}$ mice than in control mice (Figure 2E).

These observations strongly suggest that periarticular chondrocyte differentiation (periarticular to columnar) is accelerated in $I h b-B g$ mice and that this acceleration may contribute to the elongation of the growth plate.

Effect of heterozygosity of PTHrP on the length of the growth plate with upregulated Ihb action. In Ost-Cre:PPRfl- and Ibh-Bg mice, longitudinal expansion of the growth plate is primarily caused by an increase in the length of the columnar region. In these mouse models, since the rate of columnar cell proliferation was similar to that in control mice (Figure 2, D and E), the expansion of the growth plate could be due to an increase of periarticular to columnar differentiation, a decrease of columnar to hypertrophic differentiation, or both. In the mouse models with upregulation of Ihh action in the periarticular region, this increase of Ihh led to an increase in PTHrP expression. Since PTHrP inhibits premature hypertrophic differentiation in the late fetal growth plate, it is possible that the upregulation of PTHrP in the present mouse models suppresses hypertrophic differentiation and thereby contributes to the lengthening of the columnar region.

To clarify the role of PTHrP upregulation in the growth plate expansion in these mouse models, we compared degrees of

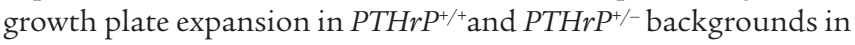
Ost-Cre:PPR $R^{f l-}$ and $I h b-B g$ mice. In the genetic background used in these experiments, PTHrP heterozygosity alone caused modest shortening of the growth plate, approximately $8 \%$ (Figure 3 , A and B). PTHrP heterozygosity caused only modest growth plate shortening of Ost-Cre:PPR $f /-$ and $\mathrm{Ihb}-\mathrm{Bg}$ mice in which the basal $P T H r P$ expression was upregulated (Figure 3, A and B). Reduction of $\mathrm{PTHrP}$ expression in $\mathrm{PTHr} \mathrm{P}^{+/-}$mice was confirmed (Figure $3 \mathrm{~B}$ and data not shown). The extent of reduction of the growth plate length in the $\mathrm{PTHr}^{+/-}$background was approximately $8 \%$ in each of these models. The observation that there was only modest growth plate shortening even in Ost-Cre:PPR ${ }^{f l-}$ and $I h b-B g$ mice, which had high basal PTHrP expression, suggests that growth plate elongation caused by Ihh overactivity may not be mediated by PTHrP upregulation alone. 


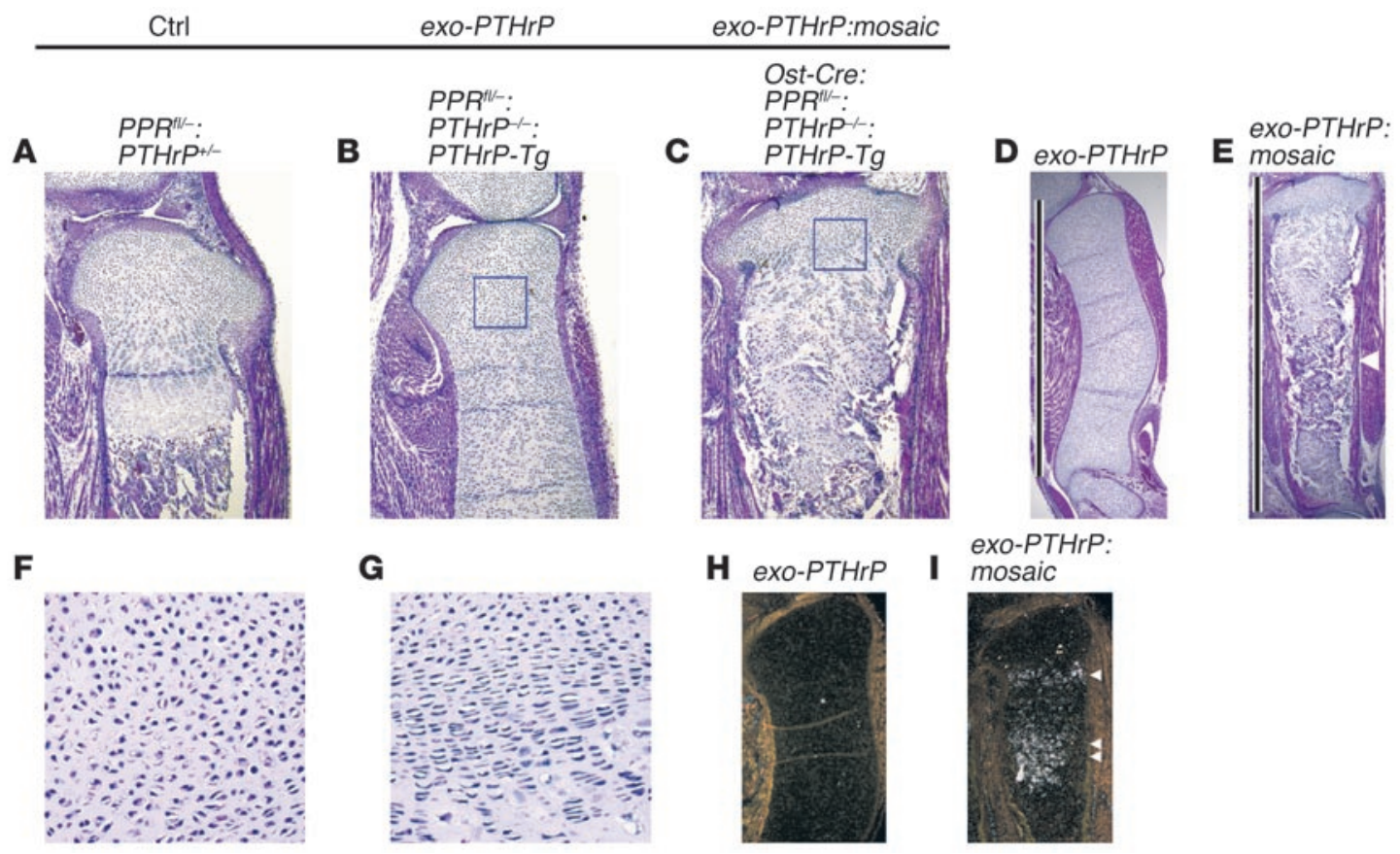

Figure 4

Ectopic hypertrophic chondrocytes induced column formation in the growth plate with exogenously controlled PTHrP. (A-C) H\&E-stained growth plates from postnatal day 0.5 (P0.5) pups with the indicated genotypes. (B and C) The endogenous PTHrP was replaced by exogenous PTHrP expressed from a transgene driven by a Col2 promoter (exo-PTHrP). (C) The PPR was ablated in a mosaic fashion in this backgound (exoPTHrP:mosaic). (D and E) Low-power views of $\mathbf{B}$ and $\mathbf{C}$. The exo-PTHrP:mosaic tibia showed a more mature appearance than the exo-PTHrP tibia with elongation of the bone (vertical lines), presence of hematopoietic marrow, and mineralized bone formation (arrowhead). $\mathbf{F}$ and $\mathbf{G}$ are magnified views of boxed areas shown in $\mathbf{B}$ and $\mathbf{C}$, respectively. Column formation was induced by the mosaic PPR ablation. (H and I) In situ hybridization for Ihh in exo-PTHrP (H) and exo-PTHrP:mosaic (I) growth plates. Mosaic PPR ablation induced Ihh expression in ectopic hypertrophic (arrowhead) and eutopic hypertrophic chondrocytes (double arrowhead).

Ectopic bypertrophic chondrocytes cause columnar cell differentiation independently of PTHrP upregulation. These observations prompted us to investigate the independent contributions of Ihh and PTHrP in regulating the columnar cell mass. Because of the mutual regulation of PTHrP and Ihh actions in the growth plate, the feedback loop between these 2 ligands had to be disrupted in order to demonstrate each molecule's distinct effect. We therefore tried to manipulate the PPR and $I b h$ genes while we maintained PTHrP signaling constant in cells in which the $P P R$ was intact.

We used the mouse model with mosaic PPR ablation in the growth plate to learn what effects ectopic hypertrophic chondrocytes had on periarticular chondrocyte differentiation under conditions of fixed PTHrP expression. To achieve this, $\mathrm{PTHrP}^{-/-}$pups were rescued by expression of an exogenous PTHrP gene controlled by a Col2 promoter (3). Mosaic PPR ablation was then induced by Cre/loxP recombination on this background. The rescued growth plates of $P$ THrP $P^{--}: P T H r P-T g$ mice (mice with exogenously provided $\mathrm{PTHrP} /$ exo-PTHrP) were similar to those of PTHrP-Tg mice with wild-type PTHrP genes (Figure 4B and data not shown): the tibia was short, and bone and bone marrow formation were suppressed. This rescued growth plate lacked hypertrophic chondrocytes and columnar cells. When mosaic Cre/loxP recombination was induced on the floxed PPR gene (exo-PTHrP:mosaic), ectopically differentiated hypertrophic chondrocytes appeared (Figure 4C). These ectopic hypertrophic chondrocytes were accompanied by an increase in the length of the tibia, by bone and marrow formation (Figure 4, $\mathrm{D}$ and $\mathrm{E}$ ), and by column formation (Figure 4, F and G). The ectopic hypertrophic chondrocytes expressed Ihb (Figure 4, H and I). Interestingly, mosaic PPR ablation stimulated eutopic hypertrophic differentiation as well (Figure 4C). This was confirmed by eutopic $I h b$ mRNA (Figure 4I) and ColX mRNA expression (data not shown). This result indicates that ectopic hypertrophic chondrocytes that express Ihh induce columnar chondrocyte differentiation independently of upregulation of PTHrP and stimulate subsequent differentiation of chondrocyte with intact PPRs toward hypertrophy.

Hedgehog directly stimulates differentiation of periarticular chondrocytes into columnar chondrocytes. In order to further determine whether Ihh directly acts on periarticular chondrocytes to stimulate differentiation into columnar chondrocytes, we used primary chondrocytes from E18.5 PTHrP-Tg tibiae that comprised almost exclusively cells with characteristics of periarticular chondrocytes (See Figure 4B). We used bagpipe homeobox gene 1 homolog (Bapx1) expression as a marker of a gene preferentially expressed in columnar chondrocytes. Bapx 1 is predominantly expressed in the columnar region of wild-type mice (Figure 5A) whereas PTHrP-Tg tibiae lack columnar chondrocytes that express Bapx1 (Figure 5B). Primary chondrocytes from $\mathrm{PTHrP}$-Tg embryos were treated with recombinant sonic hedgehog (Shh) to stimulate hedgehog signaling in these cells in vitro. Treatment with Shh significantly increased Bapx1 expression (Figure 5C), suggesting a direct stimulatory effect of hedgehog signaling on differentiation of periarticular chondrocytes. A similar result was also obtained in an in vivo model in which Ihh was overexpressed in the growth plate overexpressing PTHrP. Compared with the 
A

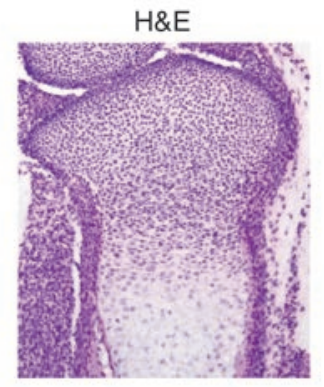

B
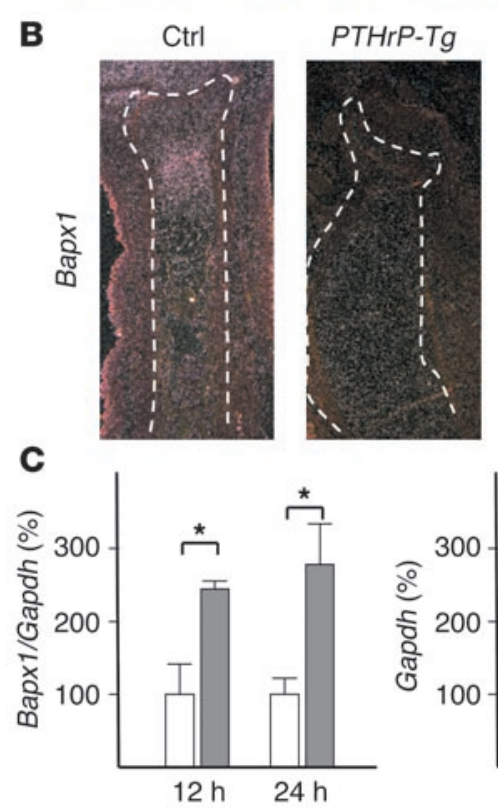

PTHrP-Tg:

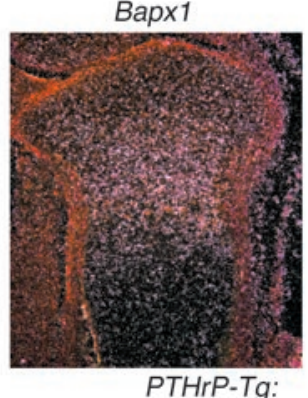

Ihh-Bg
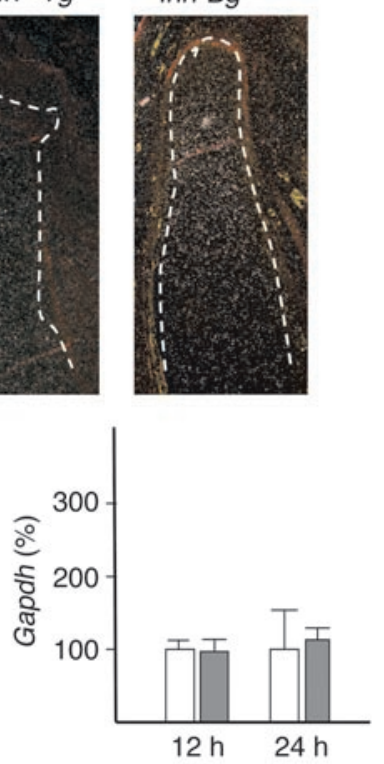

PTHrP-Tg littermate, which had few Bapx1-expressing cells, the I hh-Bg:PTHrP-Tg compound mouse showed the appearance of cells expressing Bapx1 (Figure 5B).

Ihb increases the length of the columnar region independently of PTHrP. To directly assess the role of Ihh in regulating the length of the columnar region separately from PTHrP action, we overexpressed Ihh in the growth plate in which PTHrP signaling was maintained so that it remained constant. To achieve Ihh-independent PTHrP signaling in chondrocytes, $c a P P R$ was expressed under the control of a Col2 promoter in the PTHrP-null background ( $\mathrm{caPPR}: \mathrm{PTHrP} \mathrm{P}^{-1-}$ ) constant growth plate PPR signaling [caPPR:PTHrP $\left.\left.P^{-/-} / c-P P R\right]\right)$. In this setting, PPR signaling is maintained solely by the basal activity of the $c a P P R$ transgene. The growth plate of the distal tibia of $c-P P R$ mice at E17.5 showed relatively normal appearance except for mild shortening of the columnar region and delayed blood vessel invasion (Figure 6A). Overexpression of Ihh increased the length of the columnar region even in this background with constant PPR signaling (c-PPR:Ibh-Bg) (Figure 5, A and B). Since the expression level of caPPR was too low to enable direct detection by in situ hybridization (data not shown), we examined the expression level of the endogenous Col2 mRNA (Col2/Col2a1, MGI), which was expected to correlate with the $C a P P R$ expression level. We found comparable levels of Col2 expression in c-PPR and c-PPR:Ihb-Bg mice (Figure 6B). Elongation of the columnar region was confirmed by the expansion of the domain expressing Bapx1, a gene encoding a transcription factor expressed in columnar cells (Figure 6B). Thus, independently of PTHrP, Ihh regulates the length of the columnar region.

\section{Figure 5}

Hedgehog overactivation in periarticular chondrocytes stimulates differentiation into columnar chondrocytes. (A) Bapx1 is expressed predominantly in columnar chondrocytes. H\&E staining and in situ hybridization for Bapx1 were performed on sections of the proximal tibial growth plate of an E16.5 wild-type mouse embryo. (B) Overexpression of Ihh in PTHrP-expressing cartilage induces Bapx1expressing cells. In contrast, the PTHrP-Tg tibia, comprising almost exclusively periarticular chondrocytes, lacks the Bapx1 domain. In situ hybridization was performed on distal tibiae of E16.5 embryos. Dotted lines indicate bone contours. (C) Induction of Bapx1 after Shh treatment. Chondrocytes were isolated from E18.5 PTHrP-Tg tibiae, comprising almost exclusively periarticular chondrocytes, dispersed, and cultured in vitro with or without stimulation with $1 \mathrm{mg} / \mathrm{ml}$ recombinant Shh for indicated periods. The expression of Bapx1 mRNA and Gapdh mRNA was assessed by quantitative RT-PCR. The expression of Bapx1 mRNA, a marker for columnar chondrocytes, was significantly increased after stimulation with recombinant Shh, whereas Gapdh mRNA was unchanged. The expression level of Bapx1 mRNA was normalized to that of Gapdh mRNA. Expression levels are shown as relative to mean values of control $(100 \%)$. White bars and gray bars represent control and Shh-treated cells, respectively. Standard deviations are shown. $n=3$ in each group; ${ }^{*} P<0.01$.

\section{Discussion}

In the growth plate, chondrocytes go through sequential differentiation steps. Mechanisms regulating each step are poorly understood. Differentiation of round periarticular chondrocytes into flat, column-forming columnar chondrocytes is an important step in the longitudinal growth of bones since column formation provides a clear asymmetry in the bone that allows increase in the length of the bone without proportional increase in width. Further, chondrocytes in the columnar region generally proliferate more vigorously than periarticular chondrocytes. In the present study, we provide evidence that Ihh regulates this step in a PTHrP-independent fashion.

Changes in levels of Ihh action in multiple models have demonstrated the role of Ihh in determining the length of the growth plate. First, expression of Ihh abnormally close to the periarticular region led to growth plate elongation in previously reported chimeric and mosaic growth plates $(13,14)$. This elongation is dependent on the ectopic expression of Ihh in these models; the expansion did not occur with removal of ectopic Ihh expression in chimeric growth plates comprising wild-type and $P P R^{-/-}: I h h^{-/-}$double null cells (12) or in the growth plate with double mosaic ablation of the PPR and Ihh genes in the present study. Second, we observed that Ihh heterozygosity reduced the degree of growth plate elongation in mice with mosaic PPR ablation. This effect of Ihh heterozygosity was also observed in PPR-hypomorphic mice (14), in which Ihh expression was also increased (data not shown). This is consistent with our previously proposed model that upregulation of Ihh action contributed to the growth plate phenotypes in these mice. Third, when Ihb was overexpressed, growth plate elongation occurred. These data demonstrate the causative role of the upregulated Ihh action in the growth plate expansion in these mice.

We previously showed a correlation between Ihh action in periarticular chondrocytes and the rate of differentiation of periarticular to columnar chondrocytes in several mouse models with PPR abnormalities (14). The acceleration of differentiation of periarticular to columnar chondrocytes was deduced from the 
A $c-P P R$ c-PPR:Ihh-Bg

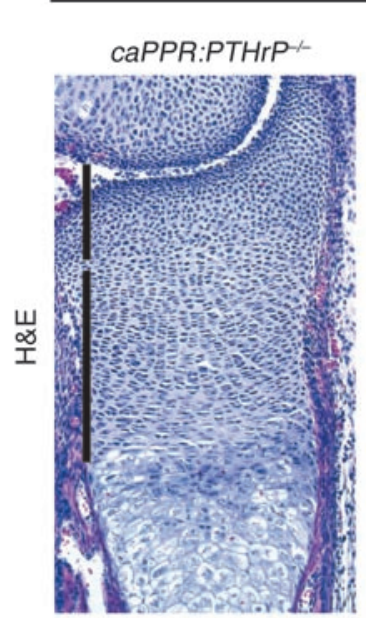

CaPPR:PTHrP-' GAL4:UAS-Ihh

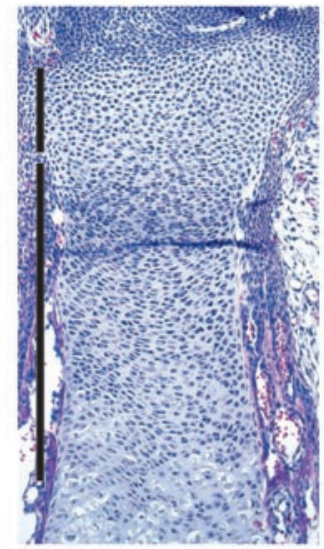

B
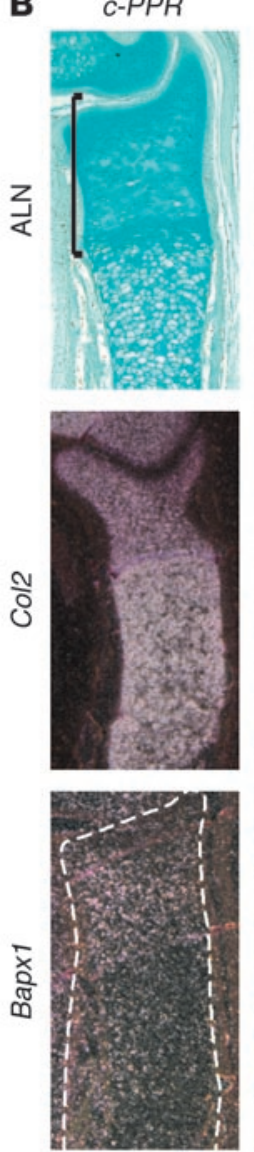

c-PPR:Ihh-Bg
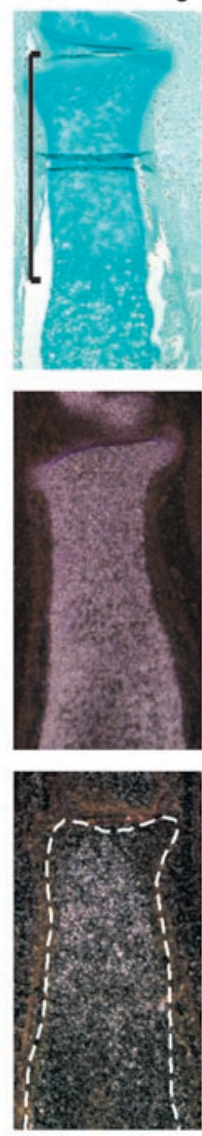

\section{Figure 6}

Growth plate elongation was induced by Ihh overexpression without upregulation of PTHrP. PTHrP-/mice were rescued by transgenic expression of caPPR to achieve Ihhindependent PPR signaling (c-PPR). Ihh was overexpressed in the growth plate with constantly maintained PPR signaling (c-PPR:Ihh-Bg). (A) H\&Estained growth plates of the distal tibia of caPPR:PTHrP-/- $(c-P P R)$ and caPPR:PTHrP-1-:GAL4:UAS-Ihh (c-PPR:Ihh-Bg). Vertical lines represent the periarticular and columnar regions. (B) Alcian blue staining and in situ hybridization assay. Col2 expression was comparable between $c-P P R$ and $c-P P R: I h h-B g$ mice. Expansion of the columnar region in $c-P P R: I h h-B g$ mice was confirmed by Bapx1 expression. Brackets indicate the proliferating zone (the periarticular and columnar regions). Dashed lines indicate bone contours. finding that, despite an increase in BrdU-labeling ratio in the periarticular region, the size of the periarticular region was smaller in these mice. We hoped to see these changes partially reversed by Ihh heterozygosity. As expected, Ihh heterozygosity in these models with upregulation of Ihh action had dramatic effects on the length of the growth plate. Because we could not detect changes in BrdU labeling associated with Ihh heterozygosity, we could not determine whether there were changes in periarticular chondrocyte differentiation. In $I h b-B g$ mice overexpressing Ihh in chondrocytes, however, we were able to detect the contrasting changes in the BrdU-labeling ratio and the number of periarticular chondrocytes at multiple developmental stages. I hb overexpression caused a decrease in cell number despite an increase in proliferation in the periarticular region whereas it caused an increase in cell number without an increase in proliferation in the columnar region. This strongly suggests that $I h b$ overexpression stimulates periarticular chondrocyte differentiation.

The relationship between hedgehog signaling and cell proliferation has been shown in various organisms in various contexts; hedgehog signaling promotes cell division and cell growth directly through $\mathrm{N}$-myc $(16,17)$, cyclin D and E expression $(18,19)$, and secondary molecules such as Wnts $(20,21)$ and IGF2 $(22)$. In the mouse growth plate, increased hedgehog signaling was associated with increases in chondrocyte proliferation in zone 1 (corresponding to the periarticular region) and an increase in cyclin $\mathrm{D}$ expression (9). However, in our models, increases in Ihh action are associated not only with increases in proliferation of periarticular chondrocytes but also with decreases in the size of the periarticular region. This suggests that stimulation of periarticular into columnar differentiation in these models is not a mere passive consequence of the increase in proliferation of the periarticular chondrocytes but is independent of the proliferative effect of Ihh.

Hedgehog overactivation in the context of PTHrP overexpression stimulated expression of BapX1, used as a marker for columnar chondrocytes, in vivo and in vitro. Since tibiae of PTHrP-Tg mice at the ages used in the present study are comprised almost solely of periarticular chondrocytes and devoid of both columnar chondrocytes and hypertrophic chondrocytes, this result is consistent with the hypothesis that hedgehog signaling in periarticular chondrocytes directly stimulates their differentiation into columnar chondrocytes. This is also consistent with the finding from our previous study of cartilage-specific PPR ablation, in which increased Ihh action in periarticular chondrocytes was associated with stimulation of chondrocyte differentiation even though few columnar chondrocytes were found in these bones (14). These results, taken together, suggest that Ihh can act directly on periarticular chondrocytes to accelerate their further differentiation.

The precise mechanisms whereby chondrocytes form stacks of flat cells in orderly arrays are incompletely understood. Studies of mice missing $\beta 1$ integrin in chondrocytes have shown that this integrin is required for the formation of flat chondrocytes but also that such chondrocytes still exhibit features that distinguish them from periarticular chondrocytes (23). This finding 


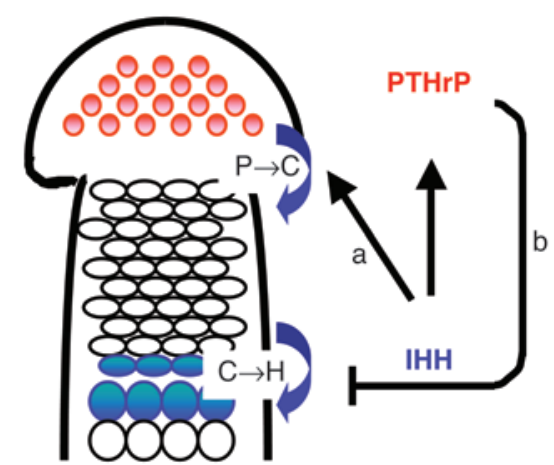

Figure 7

Model for the regulation of growth plate chondrocyte differentiation by Ihh and PTHrP at distinct steps. In addition to regulating PTHrP expression and chondrocyte proliferation, Ihh directly acts on periarticular chondrocytes to stimulate their differentiation to columnar chondrocytes (a), which regulates the length of the columnar region independently of PTHrP. PTHrP, on the other hand, is required for prevention of premature differentiation of chondrocytes into prehypertrophic and hypertrophic chondrocytes, and thereby suppresses premature expression of Ihh (b). $\mathrm{P} \rightarrow \mathrm{C}$, differentiation of periarticular chondrocytes into columnar chondrocytes; $\mathrm{C} \rightarrow \mathrm{H}$, differentiation of columnar chondrocytes into hypertrophic chondrocytes.

demonstrates that changes in the cell attachment machinery are included in the multiple changes needed for functional maturation of columnar chondrocytes.

The length of the columnar region is determined by the rate of differentiation of periarticular into columnar chondrocytes (entrance into the pool of columnar chondrocytes), the rate of columnar cell proliferation, and the rate of differentiation of columnar to hypertrophic chondrocytes (exit from the pool of columnar chondrocytes). The latter process appears to be regulated by multiple signaling molecules, including PTHrP. PTHrP, regulated by $\mathrm{Ihh}$, is crucial for normal chondrocyte differentiation. PTHrP-null mice exhibit premature terminal differentiation (1). Further, $P P R$-null and $P P R$-hypomorphic mice show acceleration of hypertrophic differentiation $(2,14)$. In contrast, overexpression of PTHrP or a constitutively active PPR suppresses the initial differentiation of hypertrophic chondrocytes $(3,4)$ (Figure 4). These findings suggest that PTHrP signaling regulates differentiation of columnar to hypertrophic chondrocytes in late fetal and perinatal growth plates, thereby regulating columnar chondrocyte mass. In this model, the length of the columnar region is determined by the stimulatory effect of Ihh on periarticular chondrocyte differentiation and the inhibitory effect of PTHrP on hypertrophic differentiation. However, the findings that PTHrP heterozygosity had only modest effects on growth plates with upregulated Ihh action and that the growth plate elongation caused by Ihh overexpression did not require upregulation of PTHrP suggest that Ihh substantially regulates the length of the columnar region independently of PTHrP.

From these observations, we propose that Ihh directly acts on periarticular chondrocytes to stimulate their differentiation to columnar chondrocytes to regulate the length of late fetal growth plates independently of PTHrP. PTHrP, on the other hand, is required for prevention of premature differentiation of chondrocytes into hypertrophic chondrocytes and thereby suppresses premature expression of Ihh (Figure 7).

\section{Methods}

Mice. PTHrP- and PPR-knockout mutant mice were described previously $(1,2)$. Col2-PTHrP and Col2-caPPR transgenic mice were described before (3, 4). I hhnull mutant and Col2-GAL4 transgenic mice were previously reported $(8,9)$. UAS-Ihh mice were newly generated by pronuclear injection using a transgene construct similar to that described in a previous report (9). Ost-Cre transgenic mice were described previously (14). PPR-floxed-neo mice (14) were crossed to enhanced FLP recombinase (flpe) transgenic mice (24) to remove the FLP recombination target-flanked (frt-flanked) neo cassette. All the $P P R$-floxed mice used in this study were neo-free. Neo-free PPR-floxed mice expressed the same amount of PPR mRNA as wild-type littermates as determined by Northern blot analysis using kidney RNA and developed normally (data not shown). I $h b$ floxed mice in which the Ihh gene contains loxP sites placed in the $5^{\prime}$ leader sequence of the first exon and in the second intron will be described elsewhere. Briefly, an frt-flanked neomycin resistant genethymidine kinase gene (neo-tk) cassette with a loxP sequence was introduced into the first intron of the Ihh gene, and another loxP site was introduced into the $5^{\prime}$ leader sequence of the first exon by homologous recombination. ES cells resistant to G418 were selected, and homologous recombination was confirmed. The initial targeting event created the floxed-neo-tk allele. ES cells were subsequently transfected with an flpe expression plasmid (25) to remove the neo-th cassette. After negative selection using gancyclovir, ES cells resistant to gancyclovir were cloned, documented to be missing the neo-th cassette by Southern blotting, and subjected to blastocyst injection to generate chimeric mice. Male chimeric mice exhibiting more than $90 \%$ chimerism were crossed to C57BL/ 6 females to obtain F1 mice.

Genotyping of mice was performed by PCR as described in the original as well as related articles except that for $I h b$-floxed allele, $P T H r P$-transgene, PTHrP-null and wild-type alleles, and Col2-caPPR-transgenic mice $(4,8,9,14)$. For genotyping the floxed thh gene, primers A: $5^{\prime}$-GATAACATGCTCTTACGAAGCTCT-3'; and B: 5'-AGCACCTTTTTTCTCGACTGCCTG-3' were used to amplify the region containing frt and loxP sequences. The floxed allele and wild-type allele generated 380-bp-long and 300-bp-long PCR products, respectively. The Col2-PTHrP transgene was determined by amplifying the $b G H$ polyA sequence included in the transgene using primers A: 5'-GGACCTAGAGGAAGGCATCCAAAC-3'; and B: 5'-TTGCTGTAGGTCTGCTTGAAGATC-3'. PCR products were approximately 300-bp long. The PTHrP-null allele was determined by PCR using specific primers A: 5'-CTTATAATCCCAGCATCTGAGAGGC-3'; and B: 5'-GATCGCCTTCTATCGCCTTCTTGAC- $3^{\prime}$. The PTHrP wild-type allele was determined using primers A: 5'-GCTACTGCATGACAAGGGCAAGTCC-3'; and B: $5^{\prime}$-GAGCCCTGCTGAACACAGTGAACAG-3'. A sequence specific to the Col2-caPPR transgene was amplified by PCR using primers A: $5^{\prime}$-TAACCATGTTCATGCCTTCTTC-3'; and B: 5'-GTCATAAATGTAGTCCGGA-3'.

Mice were primarily kept in mixed genetic backgrounds with dominance of the C57BL/6. Procedures that involved mice were approved by the Institutional Animal Care and Use Committee, Subcommittee on Research Animal Care, at Massachusetts General Hospital.

Histology and in situ bybridization. Tissues were fixed in $10 \%$ formalin/ PBS solution at $4{ }^{\circ} \mathrm{C}$, processed, embedded in paraffin, and sectioned. Sections were stained with H\&E or Alcian blue. In situ hybridization was performed as described previously (26) using ${ }^{35}$ S-labeled complementary riboprobes. The probes for mouse ColX, mouse Ptc, mouse Ihh, rat PTHrP, and mouse Bapx1 were kindly provided by Bjorn Olsen (Harvard Medical School), Ron Jonson (Stanford University), Benoit St-Jacques (Harvard University), Andrew Karaplis (McGill University), and Andrew Lasser (Harvard Medical School), respectively.

BrdU-labelingassay. Pregnant mice received intraperitoneal injections of $50 \mu \mathrm{g}$ $\mathrm{BrdU} / \mathrm{g}$ of body weight and were sacrificed 1 hour after injection. Tissues were dissected, fixed in $10 \%$ formalin/PBS overnight, paraffin processed, 
and sectioned at $5-\mu \mathrm{m}$ thickness. BrdU detection was performed using a BrdU Staining Kit (Zymed Laboraotries Inc.). BrdU-positive and -negative cells were counted separately in the periarticular region and in the columnar region, defined morphologically, and counts were compared with the total number of cells in the respective regions on each section. BrdU-labeling ratios were compared in littermates. At least 3 sections per mouse were counted, and at least 2 mice per genetic group were counted. Statistical analysis was done using ANOVA.

Quantitative RT-PCR. Quantitative RT-PCR was performed using the primer sets Bapx1-P1: 5'-GATGTCAGCCAGCGTTTCAG-3'; Bapx1-P2: 5'-CGCTTCTTTCGCGGTTTAGG-3'; GAPDH-P1: 5'-TGGAGTCTACTGGTGTCTTCA-3'; and GAPDH-P2: 5'-AAGCAGTTGGTGGTGCAGGAT$3^{\prime}$. Reverse transcription was performed on $1 \mu \mathrm{g}$ of total RNA and random hexamer primers using Superscript III (Invitrogen Corp.) according to the manufacturer's instructions. Quantitative PCR was performed using the QuantiTect SYBER Green PCR Kit (QIAGEN) and the DNA Engine Opticon 2 qPCR system (MJ Research Inc.) according to the manufacturers' instructions. Statistical analysis was performed by ANOVA using the computer software program Systat (SPSS Inc.).

Primary chondrocyte culture. Whole tibiae from E18.5 PTHrP transgenic mice were dissected, and soft tissues were removed using fine tweezers under a dissecting microscope. Isolated tibiae were washed with sterile PBS 3 times, incubated in a solution containing $0.25 \%$ trypsin and $0.2 \%$ EDTA at $37^{\circ} \mathrm{C}$ for 30 minutes, and then transferred into DMEM media containing $200 \mathrm{U} / \mathrm{ml}$ collagenase type II (Worthington Biochemical Corp.) after further removal of soft tissues and perichondrial layers. Tissues were incubated in a $5 \% \mathrm{CO}_{2}$ incubator for an hour. Cells were dispersed by pipetting, passed through $70-\mu \mathrm{m}$ nylon strainers, counted, and plated onto culture dish at a density of $5 \times 10^{4} / \mathrm{cm}^{2}$. Cells were cultured in DMEM containing 5\% fetal calf serum. After overnight culture, cells were treated with $1 \mu \mathrm{g} / \mathrm{ml}$ recombinant Shh (a gift from Curis Inc.) or vehicle for 12 or 24 hours. Total RNA was extracted using an RNAeasy Mini Kit (QIAGEN).

\section{Acknowledgments}

We thank David Buzak and Jennifer Paruch for technical assistance. We thank Susan Dymecki for flpe mice. We thank Shigeki Nishimori and Sylvain Provot for assistance in quantitative PCR and related reagents. This work was supported by NIH grant DK56246 (to H.M. Kronenberg and A.P. McMahon).

Received for publication January 5, 2005, and accepted in revised form April 5, 2005.

Address correspondence to: Henry M. Kronenberg, Endocrine Unit, Massachusetts General Hospital, 50 Blossom Street, Boston, Massachusetts 02114, USA. Phone: (617) 726-3966; Fax: (617) 7267543; E-mail: kronenberg.henry@mgh.harvard.edu.
1. Karaplis, A.C., et al. 1994. Lethal skeletal dysplasia from targeted disruption of the parathyroid hormone-related peptide gene. Genes Dev. 8:277-289.

2. Lanske, B., et al. 1996. PTH/PTHrP receptor in early development and Indian hedgehog-regulated bone growth. Science. 273:663-666.

3. Weir, E.C., et al. 1996. Targeted overexpression of parathyroid hormone-related peptide in chondrocytes causes chondrodysplasia and delayed endochondral bone formation. Proc. Natl. Acad. Sci. U. S. A. 93:10240-10245.

4. Schipani, E., et al. 1997. Targeted expression of constitutively active receptors for parathyroid hormone and parathyroid hormone-related peptide delays endochondral bone formation and rescues mice that lack parathyroid hormone-related peptide. Proc. Natl. Acad. Sci. U. S. A. 94:13689-13694.

5. Ingham, P.W., and McMahon, A.P. 2001. Hedgehog signaling in animal development: paradigms and principles. Genes Dev. 15:3059-3087.

6. McMahon, A.P., Ingham, P.W., and Tabin, C.J. 2003. Developmental roles and clinical significance of hedgehog signaling [review]. Curr. Top. Dev. Biol. 53:1-114.

7. Vortkamp, A., et al. 1996. Regulation of rate of cartilage differentiation by Indian hedgehog and PTH-related protein. Science. 273:613-622.

8. St-Jacques, B., Hammerschmidt, M., and McMahon, A.P. 1999. Indian hedgehog signaling regulates proliferation and differentiation of chondrocytes and is essential for bone formation. Genes Dev. 13:2072-2086.

9. Long, F., Zhang, X.M., Karp, S., Yang, Y., and McMahon, A.P. 2001. Genetic manipulation of hedgehog signaling in the endochondral skeleton reveals a direct role in the regulation of chondrocyte proliferation. Development. 128:5099-5108.

10. Karp, S.J., et al. 2000. Indian hedgehog coordinates endochondral bone growth and morphogenesis via parathyroid hormone related-protein-dependent and -independent pathways. Development. 127:543-548.

11. Kronenberg, H.M., and Chung, U. 2001. The parathyroid hormone-related protein and Indian hedgehog feedback loop in the growth plate. Novartis Found. Symp. 232:144-152; discussion 152-147.

12. Chung, U.I., Schipani, E., McMahon, A.P., and Kronenberg, H.M. 2001. Indian hedgehog couples chondrogenesis to osteogenesis in endochondral bone development. J. Clin. Invest. 107:295-304.

13. Chung, U., Lanske, B., Lee, K., Li, E., and Kronenberg, H. 1998. The parathyroid hormone/parathyroid hormone-related peptide receptor coordinates endochondral bone development by directly controlling chondrocyte differentiation. Proc. Natl. Acad. Sci. U. S. A. 95:13030-13035.

14. Kobayashi, T., et al. 2002. PTHrP and Indian hedgehog control differentiation of growth plate chondrocytes at multiple steps. Development. 129:2977-2986.

15. Yang, Y., Topol, L., Lee, H., and Wu, J. 2003. Wnt5a and Wnt5b exhibit distinct activities in coordinating chondrocyte proliferation and differentiation. Development. 130:1003-1015.

16. Kenney, A.M., Cole, M.D., and Rowitch, D.H. 2003. Nmyc upregulation by sonic hedgehog signaling promotes proliferation in developing cerebellar granule neuron precursors. Development. 130:15-28.

17. Pomeroy, S.L., et al. 2002. Prediction of central ner- vous system embryonal tumour outcome based on gene expression. Nature. 415:436-442.

18. Kenney, A.M., and Rowitch, D.H. 2000. Sonic hedgehog promotes $\mathrm{G}(1)$ cyclin expression and sustained cell cycle progression in mammalian neuronal precursors. Mol. Cell. Biol. 20:9055-9067.

19. Duman-Scheel, M., Weng, L., Xin, S., and Du, W. 2002. Hedgehog regulates cell growth and proliferation by inducing cyclin D and cyclin E. Nature. 417:299-304.

20. Bonifas, J.M., et al. 2001. Activation of expression of hedgehog target genes in basal cell carcinomas. J. Invest. Dermatol. 116:739-742.

21. Mullor, J.L., Dahmane, N., Sun, T., and Ruiz i Altaba, A. 2001. Wnt signals are targets and mediators of Gli function. Curr. Biol. 11:769-773.

22. Hahn, H., et al. 2000. Patched target Igf2 is indispensable for the formation of medulloblastoma and rhabdomyosarcoma. J. Biol. Chem. 275:28341-28344.

23. Aszodi, A., Hunziker, E.B., Brakebusch, C., and Fassler, R. 2003. Beta 1 integrins regulate chondrocyte rotation, G1 progression, and cytokinesis. Genes Dev. 17:2465-2479.

24. Farley, F.W., Soriano, P., Steffen, L.S., and Dymecki, S.M. 2000. Widespread recombinase expression using FLPeR (flipper) mice. Genesis. 28:106-110.

25. Buchholz, F., Angrand, P.O., and Stewart, A.F. 1998. Improved properties of FLP recombinase evolved by cycling mutagenesis. Nat. Biotechnol. 16:657-662.

26. Lee, K., Deeds, J., and Segre, G.V. 1995. Expression of parathyroid hormone-related peptide and its receptor messenger ribonucleic acids during fetal development of rats. Endocrinology. 136:453-463. 